\title{
Motion Blur Removal in Nonlinear Sensors
}

\author{
Tomer Faktor, Tomer Michaeli and Yonina C. Eldar \\ Department of Electrical Engineering \\ Technion - Israel Institute of Technology, Haifa, Israel 32000
}

\begin{abstract}
We address the problem of motion blur removal from an image sequence that was acquired by a sensor with nonlinear response. Motion blur removal in purely linear settings has been studied extensively in the past. In practice however, sensors exhibit nonlinearities, which also need to be compensated for. In this paper we study the problem of joint motion blur removal and nonlinearity compensation. Two naive approaches for treating this problem are to apply the inverse of the nonlinearity prior to a deblurring stage or following it. These strategies require a preliminary motion estimation stage, which may be inaccurate for complex motion fields. Moreover, even if the motion parameters are known, we provide theoretical arguments and also show through simulations that theses methods yield unsatisfactory results. In this work, we propose an efficient iterative algorithm for joint nonlinearity compensation and motion blur removal. Our approach relies on a recently developed theory for nonlinear and nonideal sampling setups. Our method does not require knowledge of the motion responsible for the blur. We show through experiments the effectiveness of our method compared with alternative approaches.
\end{abstract}

Keywords: Generalized sampling, interpolation, nonlinear sampling, motion blur, shift-invariant spaces.

\section{INTRODUCTION}

Imaging devices are prone to technological constraints, which lead to several types of degradation in the image sequences acquired by them. One type of degradation is due to the non-negligible integration time of image sensors, which leads to blurry images when objects in the scene move relative to the camera. If the motion is global and purely translational, then the blurring effect corresponds to convolving the image with some pointspread function (PSF). Motion debluring in this scenario is commonly treated in two stages. ${ }^{1}$ First, the motion PSF is estimated, either by assuming a specific motion model ${ }^{2-4}$ or a general one. ${ }^{1}$ Then, a debluring algorithm is applied. The latter stage can be carried out by any one of the vast amount of deblurring algorithms that assume knowledge of the PSF. These range from the Wiener filter ${ }^{5,6}$ to state-of-the-art approaches such as block-matching and the 3D filtering (BM3D) algorithm. ${ }^{7}$ An alternative paradigm for motion blur removal relies on blind deconvolution techniques that jointly determine the PSF and the original image. ${ }^{8-10}$

Another type of degradation in imaging systems is due to the nonlinear response of sensors such as chargecoupled devices (CCD). ${ }^{11,12}$ A common approach for dealing with this type of distortion is to apply intensity and contrast corrections in order to compensate for the nonlinearity. If the nonlinear response function is known, then this stage reduces to applying the inverse of the nonlinearity on each of the pixel values.

In this paper we address the problem of joint nonlinearity correction and motion deblurring (NCMD) from an image sequence. As mentioned above, motion blur and sensor nonlinearities are problems which have been extensively dealt with in the past. However, when both distortions appear together, they cannot be treated independently. Specifically, two naive NCMD approaches are to apply the inverse of the nonlinear response prior to or following a motion deblurring stage. As we explain in Section 3 and demonstrate in Section 6, both these naive methods lead to unsatisfactory results even if the blur parameters are known.

Further author information: (Send correspondence to T.F.)

T.F.: E-mail: tomerfa@tx.technion.ac.il, Telephone: +972-4-8294798, Fax: +972-4-8295757

T.M.: E-mail: tomermic@tx.technion.ac.il, Telephone: +972-4-8294682

Y.E.: E-mail: yonina@ee.technion.ac.il

This work was supported in part by the Israel Science Foundation under Grant no. 1081/07 and by the European Commission in the framework of the FP7 Network of Excellence in Wireless COMmunications NEWCOM++ (contract no. 216715). 


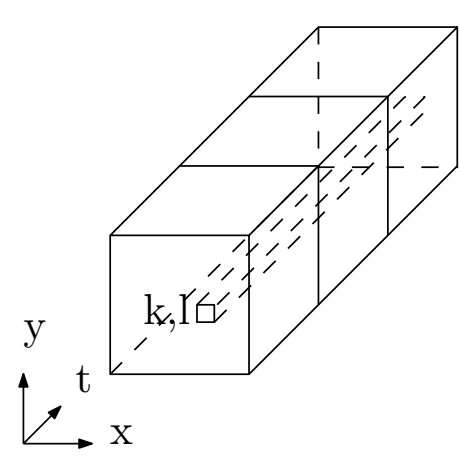

(a)

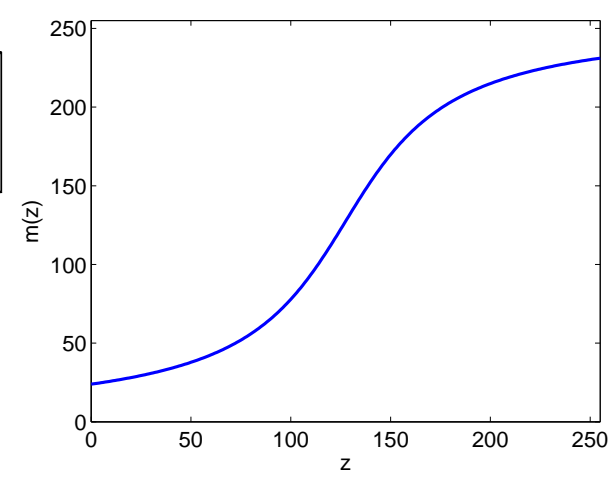

(b)

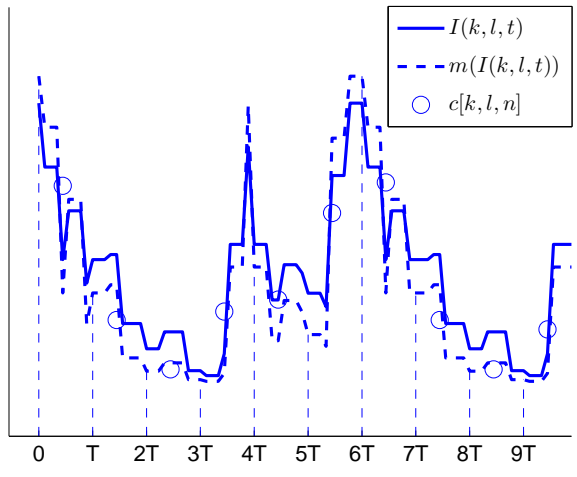

(c)

Figure 1: (a) Spatial sampling of the signal $V(x, y, t)$. (b) Nonlinear sensor response $m(z)$. (c) Temporal sampling of the signal $m(I(k, l, t))$.

The approach we present in this paper relies on a recently developed theory for nonlinear sampling. ${ }^{13}$ Specifically, the intensity values measured at a certain location in the image sensor correspond to nonindeal samples (due to the nonzero integration time) of a nonlinearly distorted version (due to the sensor's nonlinearity) of the continuous-time intensity that impinged on the corresponding location in the sensor. Thus, we propose a NCMD algorithm that recovers the continuous-time intensity corresponding to each of the pixels in the video independently. As shown in, ${ }^{13}$ this is possible under certain mild assumptions on the temporal changes in the continuous-time pixel intensities and on the nonlinearity. We propose using the iterative algorithm developed in $^{14}$ for this task. A significant advantage of our approach is that it does not require the knowledge or estimation of the motion responsible for the blur.

The paper is organized as follows. Section 2 describes the NCMD problem and presents the underlying imaging model. The naive approaches for solving the problem and their drawbacks are discussed in Section 3. Section 4 briefly presents the recently developed recovery method for the nonlinear and nonideal sampling settings, and summarizes the guarantees for its success. Our algorithm for joint motion blur removal and nonlinearity compensation is presented in Section 5. Finally, Section 6 concludes with simulation results and comparison of the performance of the different image restoration methods.

\section{PROBLEM FORMULATION}

The light intensity impinging on the image plane in an optical imaging device is a spatia-temporal signal $V(x, y, t)$ of the continuous variables $x, y$, and $t$. This signal is spatially sampled by the sensor array to yield the discretespace continuous-time signal

$$
I(k, l, t)=\iint V(x, y, t) p\left(x-l \Delta_{x}, y-k \Delta_{y}\right) d x d y
$$

Here, $k$ and $l$ are the pixel indices, $\Delta_{x}$ and $\Delta_{y}$ are the distances between adjacent pixels in the sensor array in the $x$ and $y$ directions respectively, and $p(x, y)$ is the spatial response of a single sensor cell.

Image sensors exhibit nonlinearities, such as saturation at high intensities in CCD sensors. ${ }^{11,12}$ Therefore, the voltage produced by the $(k, l)$ th sensor cell is given by $m(I(k, l, t))$, where $m(\cdot)$ is the nonlinear sensor response function. This voltage is integrated and sampled over time to produce a discrete-space discrete-time signal, which can be recorded on digital media:

$$
c[k, l, n]=\int m(I(k, l, t)) s(t-n T) d t
$$




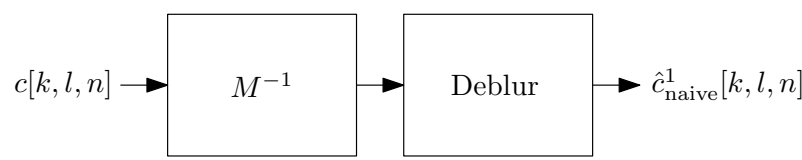

(a)

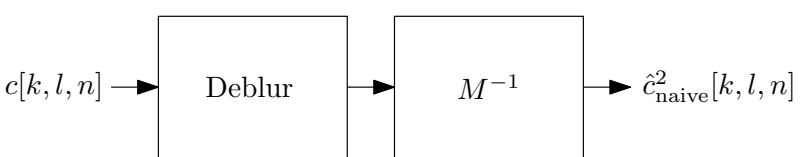

(b)

Figure 2: Block diagrams of two naive NCMD methods.

Here, $T$ denotes the the temporal sampling period and $s(-t)$ is a linear time-invariant (LTI) filter, which corresponds to the temporal integration profile.

Figure 1 demonstrates the spatial sampling, nonlinear distortion and temporal integration that yield the digital video sequence $c[k, l, n]$. The solid and dot-dashed lines in Fig. 1c show the input and measured signals respectively for a single pixel. The x-marks in this figure show the resulting pixel sequence obtained by the process (2). Due to motion in the scene during time intervals of length $T$, the filtering applied to each of the pixel signals lead to a degradation in the acquired image sequence in the form of blur.

Our goal is to restore from the acquired image sequence $c[k, l, n]$ given by $(2)$, an image sequence $\hat{c}[k, l, n]$ which is close to the input intensity $I(k, l, t)$ at times $t_{n}=n T$. This would result in a video sequence in which both the motion blur and the nonlinearity effects are removed. We note that in practical scenarios, the measurements $c[k, l, n]$ are often perturbed by the sampling device, in which case equation (2) also contains an additive noise component. However, to gain insight into the inherent difficulties in NCMD, in this paper we focus on the noise-free situation.

\section{NAIVE APPROACHES}

In this section we present two naive methods aimed at solving the NCMD problem described in Section 2. The most simple approach we can take is to treat the motion blur and the nonlinear distortion in an entirely independent fashion. Each type of image degradation will be handled separately and all that is left is to determine is which one will be treated first. Block diagrams for the two naive methods are shown in Fig. 2. The "deblur" block that appears in this figure can be implemented by any one of the algorithms that were proposed in previous works for motion-blur removal. The operator $M(\cdot)$ corresponds to applying the scalar function $m(\cdot)$ on each of the entries of its argument. The inverse operator $M^{-1}(\cdot)$ is defined similarly. Unfortunately, as appealing as these methods seem to be, they do not lead to satisfactory results. To shed light on the inherent flaws of this approach, we now examine in detail a simple example.

Consider a static scene $V_{0}(x, y)$ observed by a moving camera

$$
V(x, y, t)=V_{0}\left(x-d_{x}(t), y-d_{y}(t)\right)
$$

where the translations $d_{x}(t)$ and $d_{y}(t)$ are piecewise constant functions that take values which are integer multiples of the sensor's cell dimensions $\Delta_{x}$ and $\Delta_{y}$ respectively. Assuming without loss of generality that $d_{x}(0)=d_{y}(0)=$ 0 , we see from (1) that in this case

$$
I(k, l, t)=I\left(k-\frac{d_{y}(t)}{\Delta_{y}}, l-\frac{d_{x}(t)}{\Delta_{x}}, 0\right)
$$

for any $t$. Substituting this expression in (2) reveals that in this scenario, the digital video sequence $c[k, l, n]$ is given by

$$
c[k, l, n]=\sum_{q} \sum_{r} a_{q, r}^{n} m(I(k-q, l-r, n T))
$$

for some set of coefficients $\left\{a_{q, r}^{n}\right\}$, that are determined by the integration profile $s(t)$ and the time instances at which $d_{x}(t)$ and $d_{y}(t)$ are discontinuous. Thus, the $n$th image in the video sequence is related to the $n$th desired 
image $I(k-q, l-r, n T)$ via the matrix equation

$$
\mathbf{c}_{n}=\mathbf{A}_{n} M\left(\mathbf{i}_{n}\right),
$$

in which $\mathbf{c}_{n}$ and $\mathbf{i}_{n}$ denote the column-stack representations of $c[k, l, n]$ and $I(k, l, n T)$ respectively, and $\mathbf{A}_{n}$ is a matrix which accounts for the filtering by $a_{q, r}^{n}$. The goal of a NCMD algorithm is to recover $\mathbf{i}_{n}$ from $\mathbf{c}_{n}$. Notice that if the motion responsible for the blur is unknown, then the entries of $\mathbf{A}_{n}$ need to be estimated from the data itself. For simplicity, we will assume here that $\mathbf{A}_{n}$ is known.

In noise-free scenarios (as the one treated here), standard motion debluring approaches are designed to solve problems of the type $\mathbf{c}_{n}=\mathbf{A}_{n} \mathbf{i}_{n}$, namely without the nonlinear function $M$ appearing in (6). In this linear setting, the component of $\mathbf{i}_{n}$ perpendicular to the null-space $\mathcal{N}(\mathbf{A})$ can be recovered without error by applying the pseudo-inverse $\mathbf{A}^{\dagger}$ on the measurements $\mathbf{c}_{n}$. The component of $\mathbf{i}_{n}$ in the direction of $\mathcal{N}(\mathbf{A})$, however, is not observed. Therefore, its recovery must rely solely on available prior knowledge on $\mathbf{i}_{n}$, for example by using the fact that it corresponds to a natural image. To conclude, any reasonable deblurring method has the form

$$
\operatorname{deblur}\left(\mathbf{c}_{\mathrm{n}}\right)=\mathbf{A}^{\dagger} \mathbf{c}_{\mathrm{n}}+\mathbf{v}
$$

where $\mathbf{v} \in \mathcal{N}(\mathbf{A})^{\perp}$ is determined by the prior on $\mathbf{i}_{n}$. Using (7), the two naive methods of Fig. 2 correspond to

$$
\begin{aligned}
& \hat{\mathbf{c}}_{\text {naive }}^{1}=\operatorname{deblur}\left(\mathrm{M}^{-1}\left(\mathbf{c}_{\mathrm{n}}\right)\right)=\mathbf{A}_{\mathrm{n}}^{\dagger} \mathrm{M}^{-1}\left(\mathbf{c}_{\mathrm{n}}\right)+\mathbf{v}=\mathbf{A}_{\mathrm{n}}^{\dagger} \mathrm{M}^{-1}\left(\mathbf{A}_{\mathrm{n}} \mathrm{M}\left(\mathbf{i}_{\mathrm{n}}\right)\right)+\mathbf{v}, \\
& \hat{\mathbf{c}}_{\text {naive }}^{2}=M^{-1} \operatorname{deblur}\left(\left(\mathbf{c}_{\mathrm{n}}\right)\right)=\mathrm{M}^{-1}\left(\mathbf{A}_{\mathrm{n}}^{\dagger} \mathbf{c}_{\mathrm{n}}+\mathbf{v}\right)=\mathrm{M}^{-1}\left(\mathbf{A}_{\mathrm{n}}^{\dagger} \mathbf{A}_{\mathrm{n}} \mathrm{M}\left(\mathbf{i}_{\mathrm{n}}\right)+\mathbf{v}\right),
\end{aligned}
$$

where we used (6).

It is quite immediate to see why the first naive method (8) is expected to fail. Due to the nonlinearity of the function $m$, the operators $M^{-1}$ and $\mathbf{A}_{n}$ do not commute. Therefore, the first stage does not compensate entirely for the nonlinear distortion even if $\mathbf{A}_{n}$ is invertible, and the final result still contains distortions.

The second naive method (9) seems to be more promising at first sight. Had the matrix $\mathbf{A}_{n}$ been invertible, $\mathbf{A}_{n}^{\dagger} \mathbf{A}_{n}$ would simply be the identity operator so that $\hat{\mathbf{c}}_{\text {naive }}^{2}$ would coincide with $\mathbf{i}_{n}$. However, in the more realistic scenario of a rank-deficient matrix $\mathbf{A}_{n}, \hat{\mathbf{c}}_{\text {naive }}^{2}$ constitutes a good recovery of $\mathbf{i}_{n}$ only if the expression $\mathbf{A}_{n}^{\dagger} \mathbf{A}_{n} M\left(\mathbf{i}_{n}\right)+\mathbf{v}$ is a loyal reconstruction of $M\left(\mathbf{i}_{n}\right)$. Nevertheless, this is not the case because $\mathbf{v}$ emerges from a prior on $\mathbf{i}_{n}$ and not on $M\left(\mathbf{i}_{n}\right)$.

We conclude that from a theoretical standpoint, neither of the naive methods is appropriate for a satisfactory removal of the nonlinear distortion and motion blur. In other words, treating the nonlinear distortion and the motion blur in a separate fashion is suboptimal. In light of this understanding, we next propose a NCMD algorithm that explicitly takes into consideration the model (2).

\section{NONLINEAR AND NONIDEAL SAMPLING}

Recently, an iterative algorithm for recovering a continuous-time signal from its nonlinear and nonideal samples was proposed. ${ }^{13}$ This method was later on revised to be faster and easier to implement. ${ }^{14}$ In this section we briefly describe the recovery algorithm, ${ }^{14}$ summarize the guarantees for its success, and show how it relates to the NCMD problem.

Consider a setup in which a continuous-time signal $y(t)$ is distorted by a memoryless nonlinear mapping $m(\cdot)$ and sampled after passing through an LTI filter $s(-t)$. Notice that for any given pixel location $(k, l)$, the model (2) precisely corresponds to this setting, where $I(k, l, t), t \in \mathbb{R}$, is the continuous-time signal and $c[k, l, n]$, $n \in \mathbb{Z}$, are its nonlinear and nonideal samples. For simplicity, we will drop the pixel indices $(k, l)$ throughout this section.

We wish to produce an estimate $\hat{y}(t)$ of $y(t)$ using the recovery formula

$$
\hat{y}(t)=\sum_{n} d[n] a(t-n T)
$$




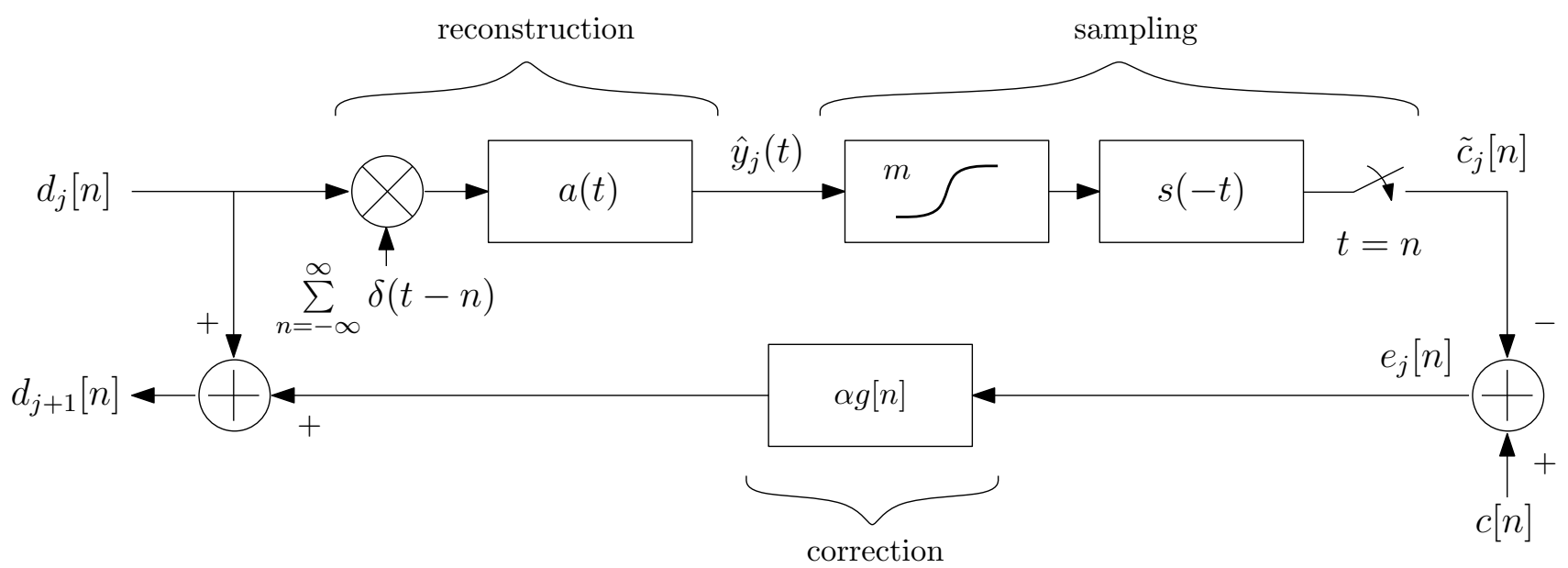

Figure 3: The $j$ th iteration of the recovery algorithm of a continuous-time signal from its nonlinear and nonideal samples. ${ }^{14}$

where $a(t)$ is a predefined reconstruction kernel and $d[n]$ is a sequence of coefficients that remains to be found.

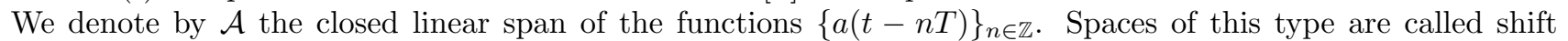
invariant (SI) and are widely used in sampling applications. ${ }^{15,16}$ Some important examples of SI spaces include the space of bandlimited signals (corresponding to $a(t)=\operatorname{sinc}(t)$ ) and splines. Similar to the reconstruction space $\mathcal{A}$, we denote by $\mathcal{S}$ the SI space generated by the sampling filter $s(t)$ and refer to it as the sampling space.

The recovery algorithm proposed $\mathrm{in}^{14}$ is designed to find a sequence of coefficients $d[n]$ such that the recovery $\hat{y}(t)$ is consistent with the original signal $y(t)$. The consistency requirement means that the nonlinear and nonideal samples of $\hat{y}(t)$ coincide with those of $y(t)$. In cases where the consistent recovery is unique, it coincides with the original signal if $y(t)$ lies in the reconstruction space $\mathcal{A}$. For signals $y(t)$ not in $\mathcal{A}$, the algorithm ${ }^{14}$ converges to $P_{\mathcal{A S}}{ }^{\perp} y$, where $P_{\mathcal{A S}}{ }^{\perp}$ is the oblique projection onto $\mathcal{A}$ along $\mathcal{S}^{\perp}$. This projection is the unique operator satisfying $P_{\mathcal{A S}} \perp y=y$ for all $y \in \mathcal{A}$ and $P_{\mathcal{A S}}{ }^{\perp} y=0$ for all $y \in \mathcal{S}^{\perp} .{ }^{16}$

One iteration of this algorithm is shown in Fig. 4. As can be seen, the expansion coefficients $d_{j}[n]$ of the $j$ th iteration are used to reconstruct an estimate $\hat{y}_{j}(t)$ of the continuous-time signal. This estimate is then distorted using $m(\cdot)$ and sampled using the filter $s(-t)$ to produce the samples $\tilde{c}_{j}[n]$. Finally, the difference between $\tilde{c}_{j}[n]$ and the true samples $c[n]$ is convolved with a filter $g[n]$, multiplied by a step-size $\alpha$, and added to $d_{j}[n]$, yielding the expansion coefficients $d_{j+1}[n]$ of the next iteration. The discrete-time Fourier transform of the correction filter $g[n]$ is given by

$$
G\left(e^{j \omega}\right)=\frac{1}{\phi_{S A}\left(e^{j \omega}\right)}
$$

Here

$$
\phi_{S A}\left(e^{j \omega}\right)=\sum_{k \in \mathbb{Z}} S^{*}(\omega-2 \pi k) A(\omega-2 \pi k)
$$

and $S(\omega)$ and $A(\omega)$ denote the continuous-time Fourier transforms of $s(t)$ and $a(t)$ respectively.

The success of the algorithm of Fig. 4 depends on the behavior of the nonlinearity and on the cosine between the sampling space $\mathcal{S}$ and reconstruction space $\mathcal{A}$, as detailed in Theorem 1 below. The cosine between two SI spaces is defined as $^{17}$

$$
\cos (\mathcal{A}, \mathcal{S})=\operatorname{ess} \inf _{\omega \in[-\pi, \pi]} \frac{\phi_{S A}\left(e^{j \omega}\right)}{\sqrt{\phi_{S S}\left(e^{j \omega}\right) \phi_{A A}\left(e^{j \omega}\right)}},
$$

where $\phi_{S A}\left(e^{j \omega}\right), \phi_{S S}\left(e^{j \omega}\right)$, and $\phi_{A A}\left(e^{j \omega}\right)$ are as in (12) with the appropriate substitution of filters $S(\omega)$ and $A(\omega)$. 
THEOREM 1. Assume that there exists a scalar $\alpha>0$ such that $\left|\phi_{S A}\left(e^{j \omega}\right)\right| \geq \alpha$ for almost every $\omega \in[-\pi, \pi]$ and that $m(\cdot)$ is differentiable almost everywhere. If $m(\cdot)$ is continuous and its derivative (when it exists) is bounded below by $q>0$ and above by $Q<\infty$, where

$$
\frac{q}{Q}>\frac{1-\cos (\mathcal{A}, \mathcal{S})}{1+\cos (\mathcal{A}, \mathcal{S})},
$$

then the iterative scheme of Fig. 4 converges to a consistent recovery $\hat{y}(t)$ of $y(t)$ given that the step size $\alpha$ satisfies

$$
\frac{1-\cos (\mathcal{A}, \mathcal{S})}{q}<\alpha<\frac{1+\cos (\mathcal{A}, \mathcal{S})}{Q} .
$$

It is important to note that consistency is a good strategy only if the samples are noise-free and the original signal is known to lie close to the reconstruction space $\mathcal{A}$. Therefore, in practice, the reconstruction kernel $a(t)$ in (10) has to be chosen in accordance with the typical behavior of the signals $y(t)$ encountered in a specific application. ${ }^{16}$ As we demonstrate in the sequel, B-splines of small degree constitute a good choice for $a(t)$, in motion blur removal applications. We comment that when the samples are noisy, the consistency approach is often modified to include a regularization term, which is aimed at suppressing the noise. ${ }^{18}$ In this paper we focus on the noise-free case and thus disregard this term.

\section{PROPOSED ALGORITHM}

Based on the algorithm presented in the previous section, we now present a simple NCMD method which operates as follows. For each pixel location $(k, l)$, the iterative algorithm of ${ }^{14}$ is applied on the sequence $c[k, l, n], n \in \mathbb{Z}$, producing a consistent approximation $\hat{I}(k, l, t), t \in \mathbb{R}$ of the continuous-time light intensity collected by the $(k, l)$ th sensor element. The recovered image sequence, is then obtained by sampling $\hat{I}(k, l, t)$ at times $t_{n}=n T$ :

$$
\hat{c}[k, l, n]=\hat{I}(k, l, n T)
$$

A pseudo-code of this approach is given in Algorithm 1 below. For a video sequence comprising $N$ frames of size $K \times L$, the complexity of the algorithm is $\mathcal{O}(K L N)$.

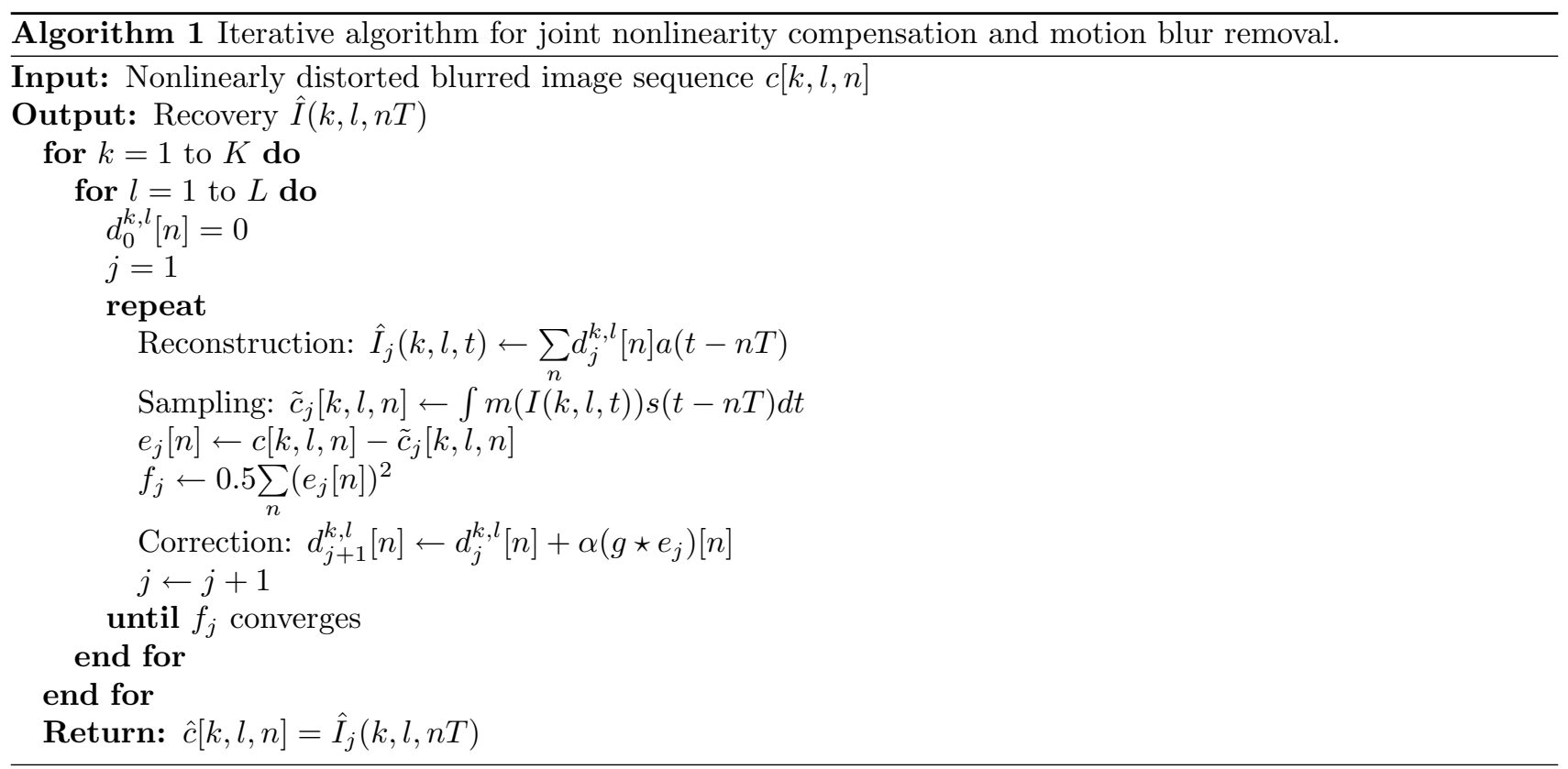

We emphasize that this algorithm does not require knowledge of the motion responsible for the blur, which is a significant advantage over the naive methods. Assuming the conditions of Theorem 1 are satisfied, the quality 


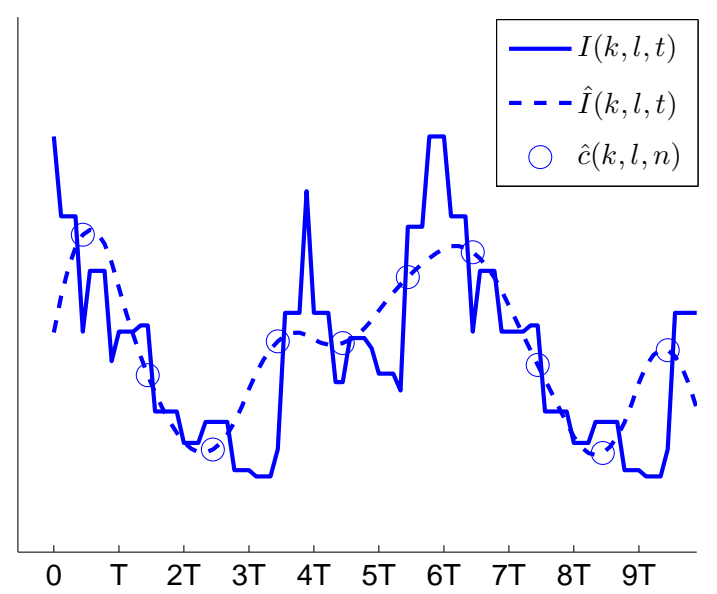

Figure 4: Iterative recovery for one pixel sequence.

of the recovery depends solely on the choice of the reconstruction kernel $a(t)$ in (10). In our experiments, we used a B-spline of degree 2. With this choice, the reconstructed signal $\hat{I}(k, l, t)$ is a spline of degree 2 . That is, $\hat{I}(k, l, t)$ is a continuously differentiable piecewise polynomial of degree 2 with breaks at integer multiples of $T$. As we show in the next section, this choice is especially adequate for textured objects experiencing small translations with respect to the camera.

Figure 4 demonstrates the recovery capability of the algorithm. In this experiment, the sampling filter $s(-t)$ is a rectangular kernel of width $T$ and the nonlinearity is given by $m(z)=\lambda \arctan \left(\gamma\left(z-z_{0}\right)\right)$, as depicted in Fig. 1b. The parameters $\lambda, \gamma, z_{0}$ were chosen to satisfy condition (14) of Theorem 1 . The solid and dashed lines correspond to the original and recovered signals respectively for a single pixel. The recovered signal is a spline of degree 2 and thus does not match the original signal, which is much more edgy. However, as we see from the o-marks in the figure, both signals are relatively close at times $t_{n}=n T$.

\section{SIMULATIONS}

We now compare the iterative algorithm that was described in Section 5 to the naive methods of Section 3 . In this experiment, we generated a movie sequence from the $512 \times 512$ Mandrill image by applying a circular global translation

$$
d_{x}(t)=\Delta_{x} \operatorname{round}\left\{\beta \cos \left(\frac{2 \pi t}{T_{\mathrm{m}}}\right)\right\}, \quad d_{y}(t)=\Delta_{y} \operatorname{round}\left\{\beta \sin \left(\frac{2 \pi t}{T_{\mathrm{m}}}\right)\right\} .
$$

This type of motion models a rattling of the camera during long exposure periods. In order to ensure that the motion is not too rapid, we set $\beta=3$ pixels, and $T_{\mathrm{m}}=6 T$, where $T$ is the sampling interval. We used the nonlinear response that appears in Fig. $1 \mathrm{~b}$ and a rectangular sampling filter $s(t)$ of width $T$. Figure 5 depicts the original Mandrill image and one of the images in the resulting degraded sequence.

For the two naive methods, we assumed that the global motion is known, so that an appropriate PSF can be provided for the deblurring stage. We used the BM3D algorithm ${ }^{7}$ for this purpose. For our iterative algorithm, we used a step size of $\alpha=0.8$, which satisfies the condition of Theorem 1 . In order to reveal the differences between the different recovery methods, we focus on patches of size $128 \times 128$ located on the mid-left side of the images. The recovered patches, along with the ground truth and degraded patches are shown in Fig. 6 . We can see from this figure, that in both naive methods the bright and dark textures look smeared. In the iterative recovery, on the other hand, the texture appears to be more detailed and with better contrast. Overall, the iterative recovery is the closest to the original image.

Note that this experiment was performed for the scenario where there is global motion in the movie and we assumed that this motion is known to us. Notwithstanding, our algorithm outperformed the naive methods. 


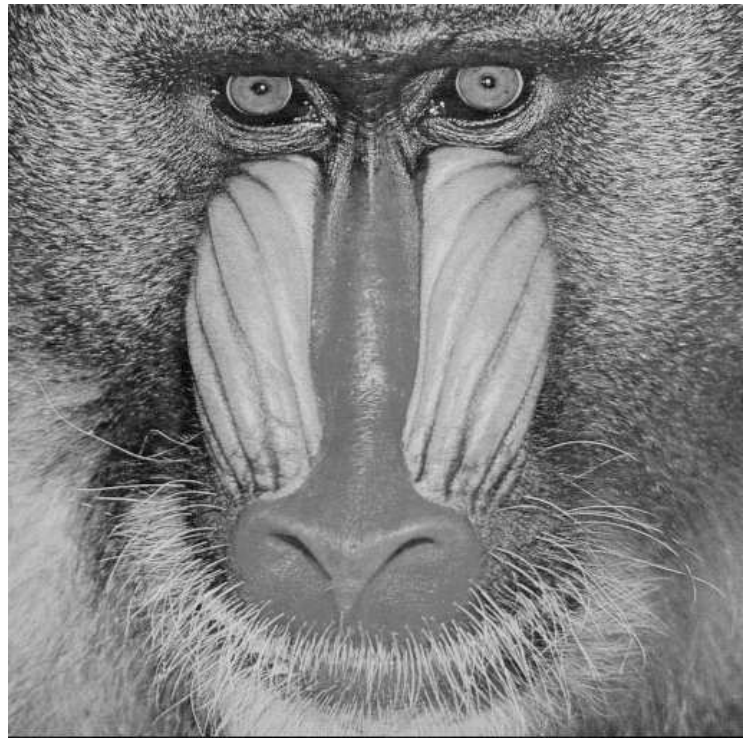

(a)

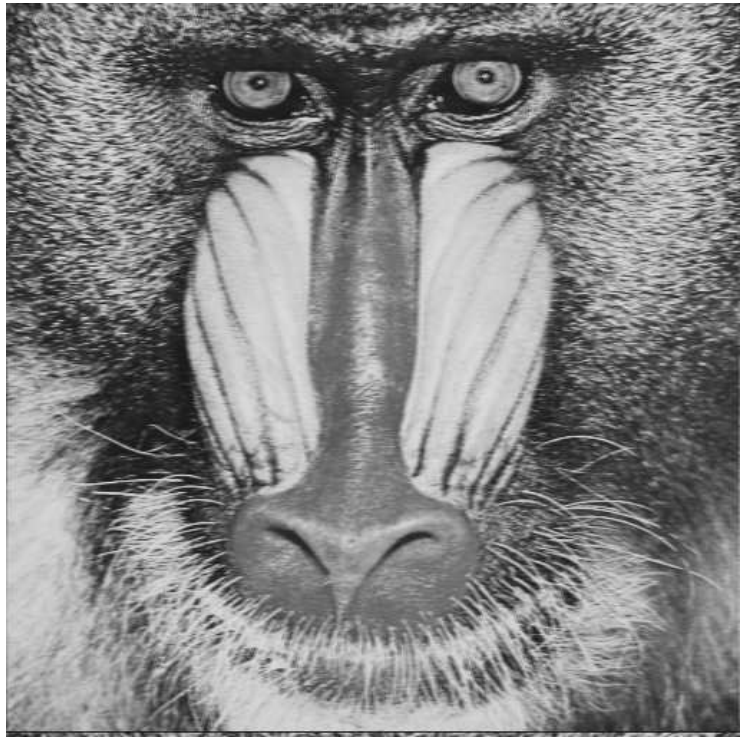

(b)

Figure 5: (a) Original Mandrill image. (b) A nonlinearly distorted motion-blurred version of the Mandrill image.

If the motion is unknown, the complexity of the naive methods is increased and the differences between the recoveries is expected to become more salient in favor of our approach.

\section{CONCLUSION}

In this paper we addressed the problem of joint motion blur removal and nonlinearity compensation of an image sequence. We presented a novel recovery algorithm that is based on the recently developed theory and methods for nonlinear and nonideal sampling. This algorithm does not require knowledge or an estimation stage of the motion responsible for the blur. This is a significant advantage over naive methods which rely on standard deblurring algorithms requiring knowledge of the blur parameters. We demonstrated that when the motion is not too rapid, our algorithm outperforms the naive methods, even in the case where we assume that the motion is known. The differences in favor of our approach are eminent especially in regions containing textures.

\section{REFERENCES}

[1] Fergus, R., Singh, B., Hertzmann, A., Roweis, S. T., and Freeman, W. T., "Removing camera shake from a single photograph," in [ACM SIGGRAPH], 787-794, ACM (2006).

[2] Fabian, R. and Malah, D., "Robust identification of motion and out-of-focus blur parameters from blurred and noisy images," Graphical Models and Image Processing 53, 403-412 (1991).

[3] Li, Q. and Yoshida, Y., "Parameter estimation and restoration for motion blurred images," IEICE Trans. Fundamentals E80-A(8), 1430-1437 (1997).

[4] Lokhande, R., Arya, K. V., and Gupta, P., "Identification of parameters and restoration of motion blurred images," Proc. ACM, 301-305 (January 2006).

[5] Lagendijk, R. L. and Biemond, J., [Hand Book of Image and Video Processing], Academic Press (2000).

[6] Gonzalez, R. C. and Woods, R. E., [Digital Image Processing], Pearson Education (2003).

[7] Dabov, K., Foi, A., and Egiazarian, K., "Image restoration by sparse 3D transform-domain collaborative filtering," Proc. SPIE Electronic Imaging, 6812-07 (January 2008).

[8] Kundur, D. and Hatzinakos, D., "A novel blind deconvolution scheme for image restoration using recursive filtering," IEEE Trans. Signal Processing 46, 375-390 (1998). 


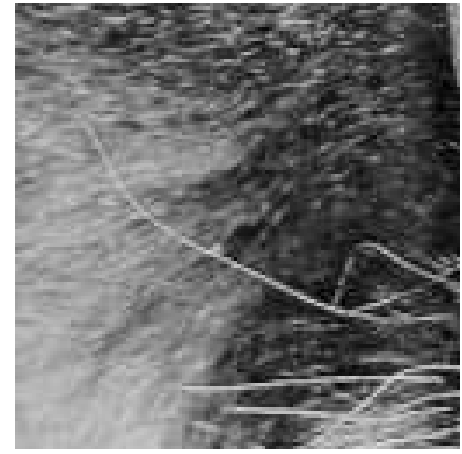

(a)

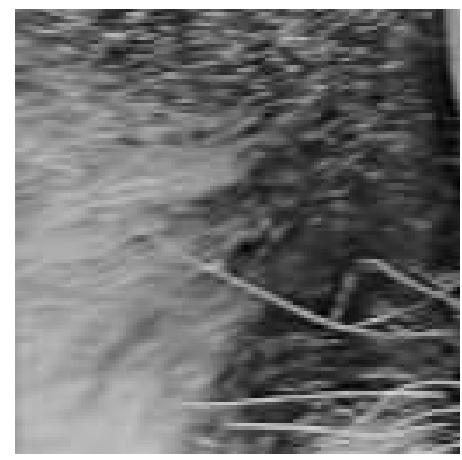

(d)

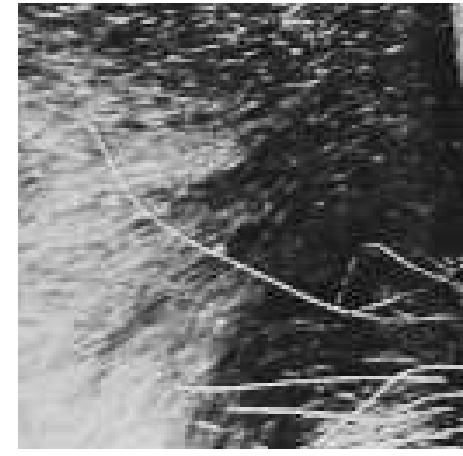

(b)

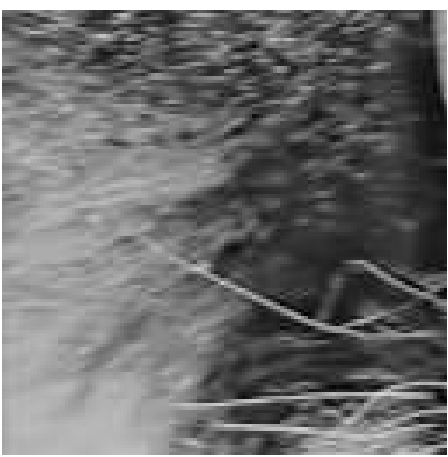

(e)

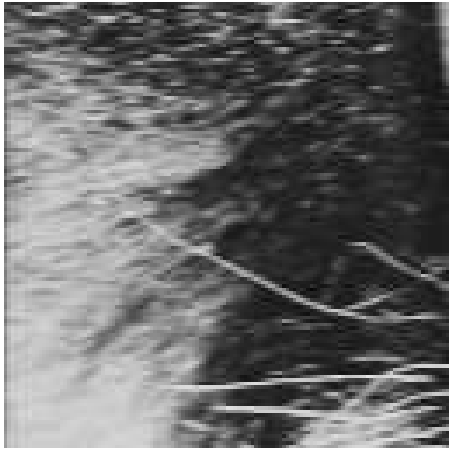

(c)

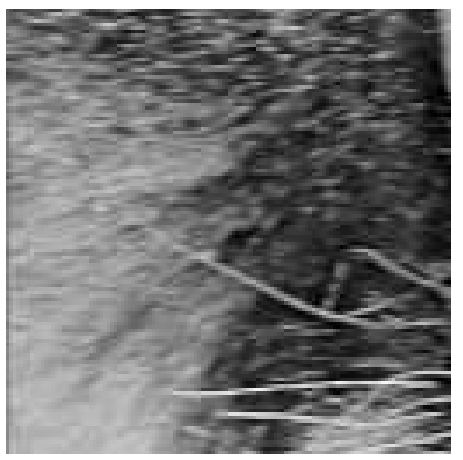

(f)

Figure 6: (a) Original image. (b) Degradation by nonlinear sensor response. (c) Degradation by nonlinear response and motion blur. (d) Recovery using the first naive method (8). (e) Recovery using the second naive method (9). (f) Recovery using our proposed iterative algorithm.

[9] Likas, A. and Galatsanos, N. P., "A variational approach for bayesian blind image deconvolution," IEEE Trans. Signal Processing 52(8), 2222-2233 (2004).

[10] Molina, R., Mateos, J., and Katsaggelos, A., "Blind deconvolution using a variational approach to parameter, image, and blur estimation," IEEE Trans. Image Processing 15(12), 3715-3727 (2006).

[11] Kawai, S., Morimoto, M., Mutoh, N., and Teranishi, N., "Photo response analysis in CCD image sensors with a VOD structure," IEEE Trans. Electron Devices 42(4), 652-655 (1995).

[12] Holst, G. C., [CCD Arrays Cameras and Displays], SPIE Optical Engineering Press, Bellingham, WA (1996).

[13] Dvorkind, T. G., Eldar, Y. C., and Matusiak, E., "Nonlinear and nonideal sampling: theory and methods," IEEE Trans. Signal Processing 56, 5874-5890 (December 2008).

[14] Faktor, T., Michaeli, T., and Eldar, Y. C., "Nonlinear and nonideal sampling revisited," to appear in IEEE Signal Processing Letters .

[15] Unser, M., "Sampling - 50 years after Shannon," Proc. IEEE 88, 569-587 (April 2000).

[16] Eldar, Y. C. and Michaeli, T., "Beyond bandlimited sampling," IEEE Signal Processing Magazine 26, 48-68 (May 2009).

[17] Unser, M. and Aldroubi, A., "A general sampling theory for nonideal acquisition devices," IEEE Trans. Signal Processing 42, 2915-2925 (November 1994).

[18] Ramani, S., Van De Ville, D., Blu, T., and Unser, M., "Nonideal sampling and regularization theory," IEEE Trans. Signal Processing 56(3), 1055-1070 (2008). 